\title{
MULTIFOCUS IMAGE FUSION USING CLOUD MODEL
}

\author{
K. Kannan \\ Department of Mechanical Engineering, Kamaraj College of Engineering and Technology, India \\ E-mail: kannan_kcet@yahoo.co.in
}

\begin{abstract}
This paper proposes a multifocus image fusion algorithm based on cloud model. First, each source images are divided into overlapping image blocks of size $(2 N+1) \times(2 N+1)$ and then the mean and entropy of every image pixels over this neighborhood window was calculated and compared in Cloud domain. The pixel with higher magnitude of the calculated image features was selected to form the fused image. The results of multifocus image fusion using this algorithm hold favorable consistency in terms of root mean square error, peak signal to noise ratio and quality index for three pairs of test images and confirm the effectiveness of the proposed algorithm.
\end{abstract}

Keywords:

Multi Focus Image Fusion, Cloud Model

\section{INTRODUCTION}

When a scene is being imaged, it is desirable to take the clear image of all objects present in the scene. Due to limited Depth of Field (DOF) of lens used in many engineering applications, it is possible to take clear image of the objects which are in focus only. The remaining objects in the scene will be out of focus. This is a major issue in many engineering applications. To have the focused image of all the objects in the scene, multi focus image fusion is needed. Multifocus image fusion is the process of combining two or more images of the same scene with different focus points to form the fused image. The objective of multi focus image fusion is to produce the fused image in which all objects are in focus. Various techniques have been reported in the literature which is classified into spatial fusion and multiscale transform fusion. In spatial fusion, the pixel values from the source images are directly manipulated to form the pixel of the fused image. It has been found that the spatial fusion methods perform well and at the same time they will reduce the contrast of features uniquely present in the source images [1]. Better results were obtained if fusion takes place at multiscale in the transform domain. In recent years, multiscale transform has been recognized as a useful approach to analyze the information content of images for the purpose of image fusion. Multiscale transform based fusion methods decomposes each input image, integrate the decompositions to construct the composite representation of the fused image. The fused image is then obtained by taking an inverse multiscale transform. This technique is more complicated to design and time consuming process to implement [2]. The key challenge of multi focus image fusion is to evaluate the sharpness of each image and then select information from the most informative sharp image to form the fused image [3]. To evaluate the sharpness of the image, this paper uses the cloud model. In this paper, two different images of the same scene with different focus points are fused using cloud model and its performance is compared with some existing methods in terms of root mean square error, peak signal to noise ratio and quality index.

The remainder of the paper is organized as follows. In section 2, a review of the necessary background required to effectively implement our algorithm is presented. The proposed algorithm is described in section 3. Section 4 presents the evaluation criteria used in this paper to evaluate the results. After that, results of the proposed algorithm are presented in section 5 . Last section presents the conclusion.

\section{CLOUD MODEL}

Fuzzy provides a method to transact the fuzziness and randomness. The commonly used method of uncertainty reasoning is based on fuzzy set theory [4]. The basis of fuzzy set theory is the membership function. The membership function is a one-point to one-point mapping from a space $U$ to the unit interval $[0,1]$. After the mapping, the uncertainty of an element belonging to the fuzzy concept becomes certain to the degree represented by a precise number. The uncertain characteristics of the original concept are not passed on to the next step of processing at all. This is the intrinsic shortcoming of the fuzzy set theory. In order to overcome this shortcoming, Dr. D. Y. Li proposed the cloud model (CM) [5]. CM is a model of the uncertainty transformation between quantitative representation and qualitative concept based on normal distribution and bell shaped membership function. Now, $\mathrm{CM}$ has been extended to two dimensional cases and explored in digital image processing applications. In specific, CM has been successfully applied to data mining [6, 8], image classification [7], image segmentation $[9,10]$ and optimization [11].

Let $U$ is a quantity domain expressed with accurate numbers and $C$ is a quality concept in $U$. If the quantity value, $x \in U$ and $x$ is a random realization of the quality concept $C$, then $\mu(x)$ is the membership degree of $x$ which lies between [0,1]. It is the random number which has the steady tendency,

$$
\mu: U \rightarrow[0,1], \forall x \in U, x \rightarrow \mu(x)
$$

The distribution of $x$ is called cloud and each $x$ is called a cloud drop [6]. The cloud can be characterized by three parameters, i.e., the expected value $E_{x}$, entropy $E_{n}$, and hyperentropy $H_{e}$ [6-11]. $E_{x}$ is the expectation of the cloud drops' distribution. It points out which drops can best represent the concept and reflects the distinguished feature of the concept. $E_{n}$ is the uncertainty measurement of the qualitative concept, which is determined by both the randomness and the fuzziness of the concept. It represents the value region in which the drop is acceptable by the concept, while reflecting the correlation of the randomness and the fuzziness of the concept. $H_{e}$ is the uncertainty measurement of $E_{n}$. Given these three characteristics, a set of cloud drops can be generated with certainty degree by the normal cloud generator CG. Each pixel in the image is the cloud drop and 
composes the cloud. These cloud drops are given input to the backward cloud generator $\mathrm{CG}^{-1}$. The outputs of $\mathrm{CG}^{-1}$ are three parameters of cloud $E_{x}, E_{n}$ and $H_{e}$. This is shown in Fig.1.

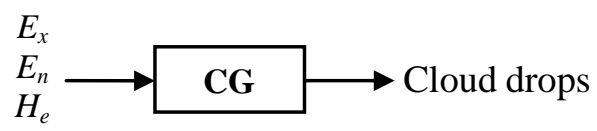

Fig.1(a). Forward Cloud Generator

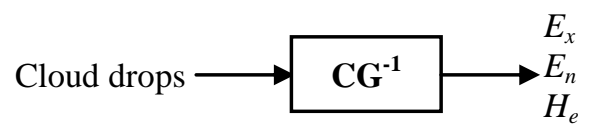

Fig.1(b). Backward Cloud Generator

According to the normal cloud generator (CG), the certainty degree of each drop is a probability distribution rather than a fixed value. It means that the certainty degree of each drop is a random value in a dynamic range. If $H_{e}$ of the cloud is 0 , then the certainty degree of each drop will change to be a fixed value. The fixed value is the expectation value of the certainty degree. In fact, the value is also the unbiased estimation for the average value of the certainty degrees in the range. All the drops and their expectations of certainty degrees can compose a curve, and the curve is the cloud expectation curve (CEC). The CEC of cameraman image is shown in the Fig.2.

\section{PROPOSED ALGORITHM}

This section discusses multifocus image fusion to form allin-focus image from two images of the same scene with different focus points. The structure of proposed methodology is shown in Fig.3. Let there are two source images $A \& B$ and $N=4$. In the first step, each source image is divided into $(2 N+1) \times(2 N+1)$ window of overlapping regions. Let $W_{i, j}^{2 N+1}$ be a window of size $(2 N+1) \times(2 N+1)$ centered at location $(i, j)$, and $x_{i, j}$ denotes the gray value of the pixel at location $(i, j)$. Then, the mean value of the window $W_{i, j}^{2 N+1}$ for each and every pixel in two source images $A$ and $B$ (denoted as $E_{x A}(i, j)$ and $\left.E_{x B}(i, j)\right)$ is calculated using the formula,

$$
E_{x}=\frac{1}{n} \sum_{x_{i+s, j+\epsilon \in} W_{i, j}^{2 N+1}} x_{i+s+t}
$$

The entropy $E_{n}$ of the pixels of source images $A$ and $B$ (denoted as $E_{n A}(i, j)$ and $E_{n B}(i, j)$ ) is calculated using the following formula,

$$
E_{n}=\sqrt{\frac{\pi}{2}} \times \frac{1}{n} \sum_{x_{i+s, j+t \in} W_{i, j}^{2 N+1}}\left|x_{i+s, j+t}-E_{x}\right|
$$

In the next step, the fused image $F$ is produced by combining two source images as

$$
F(i, j)=\left\{\begin{array}{lll}
A(i, j) & \text { if } & E_{n A}(i, j) \geq E_{n B}(i, j) \\
B(i, j) & \text { if } & E_{n B}(i, j)>E_{n A}(i, j)
\end{array}\right\}
$$

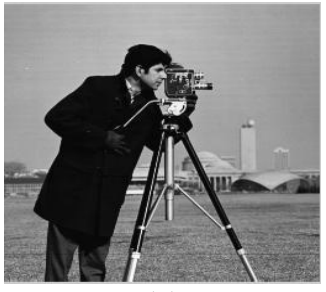

(a) (b)

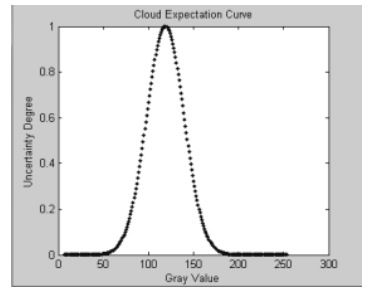

Fig.2(a). Cameraman Image, (b).CEC

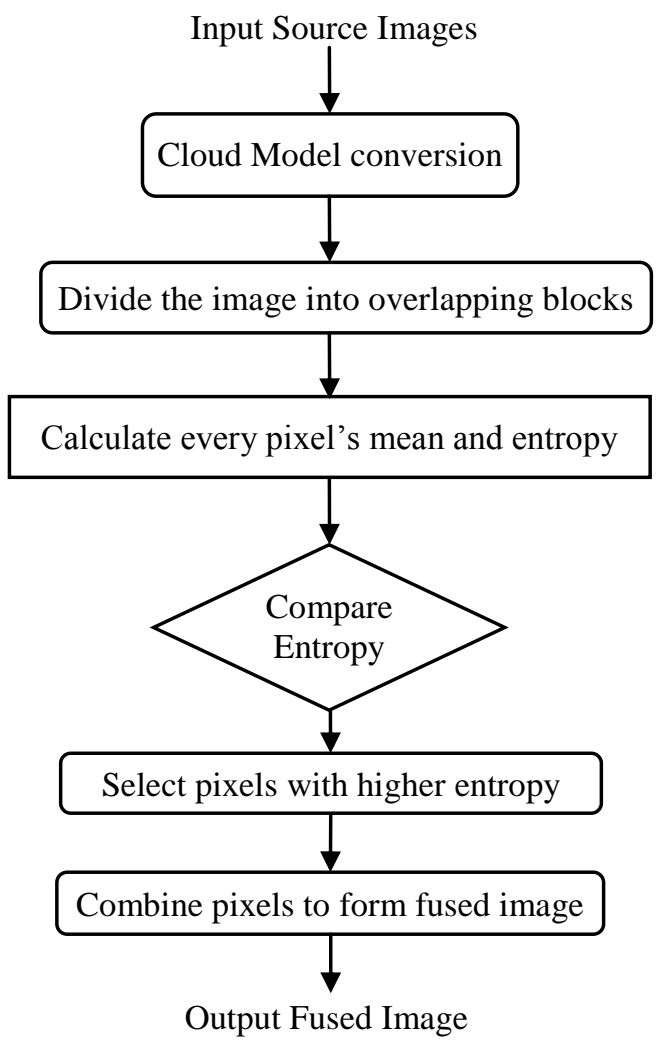

Fig.3. Architecture of the Proposed Algorithm

\section{EVALUATION CRITERIA}

The evaluation measures are used in this paper, as follows, The Root Mean Square Error (RMSE) between the reference image $R$ and fused image $F$ is given by [12],

$$
R M S E=\sqrt{\frac{\sum_{i=1}^{N} \sum_{j=1}^{N}[R(i, j)-F(i, j)]^{2}}{N^{2}}}
$$

The Peak Signal to Noise Ratio (PSNR) between the reference image $R$ and fused image $F$ is given by [12],

$$
\text { PSNR }=10 \log _{10}(255)^{2} /(\mathrm{RMSE})^{2}(\mathrm{db})
$$

Quality index of the reference image $(R)$ and fused image $(F)$ is given by [13],

$$
Q I=\frac{4 \sigma_{a b} a b}{\left(a^{2}+b^{2}\right)\left(\sigma_{a}^{2}+\sigma_{b}^{2}\right)}
$$


The maximum value $\mathrm{Q}=1$ is achieved when two images are identical, where a $\& \mathrm{~b}$ are mean of images, $\sigma_{a b}$ be covariance of $R \& F, \sigma_{a}^{2}, \sigma_{b}^{2}$ be the variance of image $R, F$.

\section{EXPERIMENTS AND RESULTS}

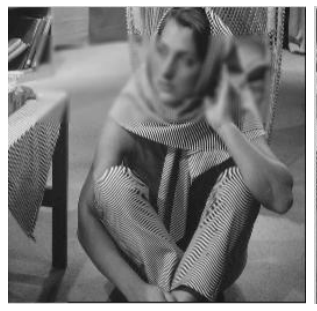

(a)

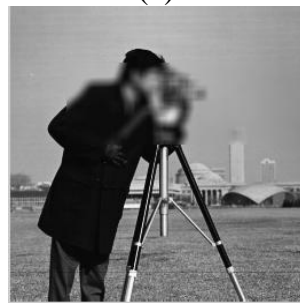

(a)

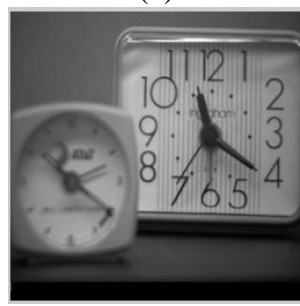

(a)

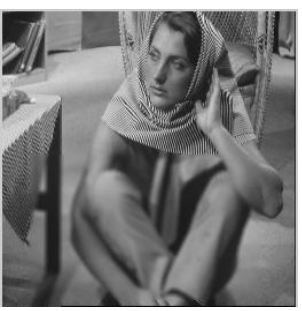

(b)

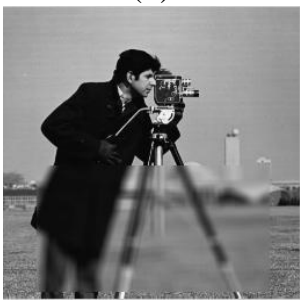

(b)

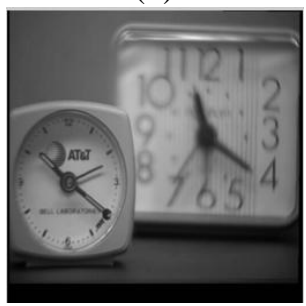

(b)

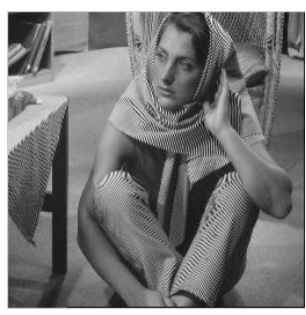

(c)

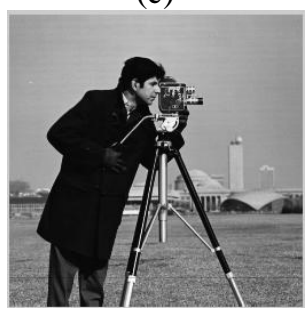

(c)

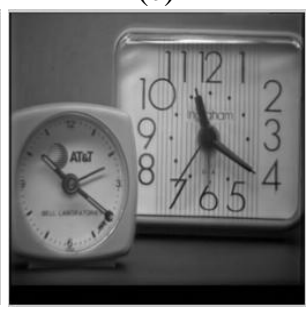

(c)
Fig.4. Row1. Barbara Image, Row2. Cameraman Image, Row3. Clock Image - (a). Source Image1, (b). Source Image2, (c). Reference Image

To verify the performance of the proposed multifocus image fusion algorithm, experiments were conducted with three pairs of image sets. Two pairs of images are artificially generated by applying blurs in different parts of the standard images namely Barbara and Cameraman images. To represent the two images of the same scene with different focus points, the clock images were used. These three pairs of test images along with the reference images are shown in Fig. 4.
Table.1. Comparison of Multifocus Image fusion using various methods

\begin{tabular}{|c|c|c|c|c|c|}
\hline \multicolumn{7}{|c|}{ Barbara } \\
\hline & $\begin{array}{c}\text { Tian- } \\
\text { 2011[2] }\end{array}$ & $\begin{array}{c}\text { Tian - } \\
\text { 2012[3] }\end{array}$ & Variance & $\begin{array}{c}\text { Energy } \\
\text { Gradient }\end{array}$ & Proposed \\
\hline RMSE & 6.826 & 0.684 & 1.790 & 0.619 & 0.586 \\
\hline PSNR & 31.447 & 51.426 & 43.072 & 52.293 & 52.768 \\
\hline QI & 0.989 & 0.999 & 0.999 & 0.999 & 0.999 \\
\hline \multicolumn{7}{|c|}{ Cameraman } \\
\hline RMSE & 10.683 & 2.606 & 2.781 & 1.846 & 1.443 \\
\hline PSNR & 27.556 & 39.808 & 39.244 & 42.803 & 44.941 \\
\hline QI & 0.984 & 0.999 & 0.999 & 0.999 & 0.999 \\
\hline \multicolumn{7}{|c|}{ Clock } \\
\hline RMSE & 7.701 & 6.043 & 6.589 & 6.011 & 5.932 \\
\hline PSNR & 30.399 & 32.504 & 31.754 & 32.550 & 32.666 \\
\hline QI & 0.988 & 0.993 & 0.991 & 0.993 & 0.993 \\
\hline
\end{tabular}

These test images are subjected to $\mathrm{CM}$ filtering and combined to form the fused image. To evaluate the performance, the fused image is compared with the reference image quantity performance metrics such as RMSE, PSNR and QI are calculated. To evaluate the efficiency of the proposed algorithm, it is compared with fusion using variance, energy of gradient, bilateral sharpness criterion [2] and wavelet based statistical sharpness measure [3]. The results are shown in Fig.5 and tabulated in Table.1. From the results, it is inferred that the proposed method provides better results consistently than the other methods due to stability of characteristics of CM.

\section{CONCLUSION}

This work presents a computationally efficient method designed for multifocus image fusion algorithm. Firstly, the source images are divided into $(2 N+1) \times(2 N+1)$ window of overlapping image blocks. Then, the mean and entropy of each pixel of every source image was calculated over this block, and the fused image is formed by combining the pixels from the source image with higher magnitude. The experimental fusion results hold favorable consistency in terms of root mean square error, peak signal to noise ratio and quality index for three pairs of test images and confirm the effectiveness of the proposed algorithm.

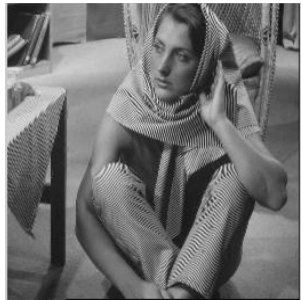

(a)

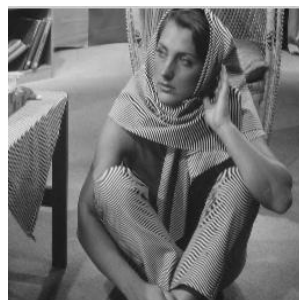

(b)

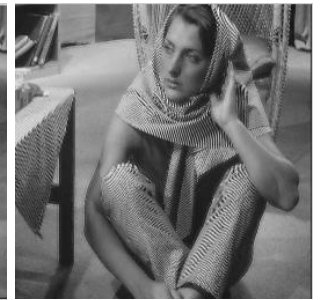

(c)

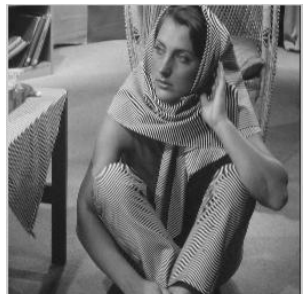

(d)

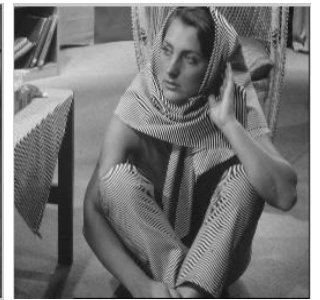

(e)

Fig.5. Experimental results of Image fusion for Barbara Image - Fused Image using (a). Variance, (b). Energy gradient, (c). Bilateral sharpness, (d). Wavelet based statistical sharpness, (e). Proposed method 


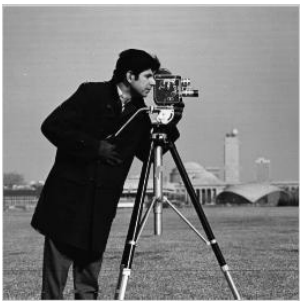

(a)

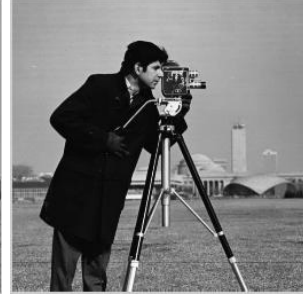

(b)

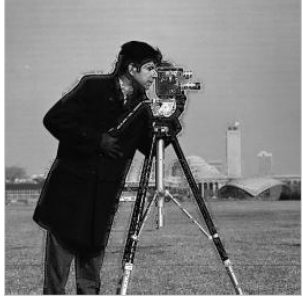

(c)

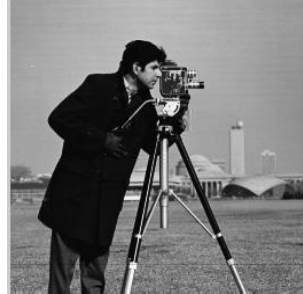

(d)

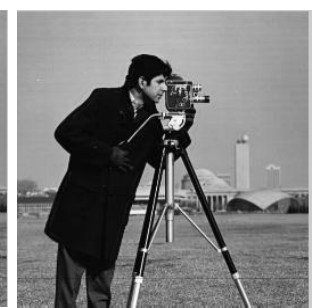

(e)

Fig.6. Experimental results of Image fusion for Cameraman Image - Fused Image using (a). Variance, (b). Energy gradient, (c). Bilateral sharpness, (d). Wavelet based statistical sharpness, (e). Proposed method

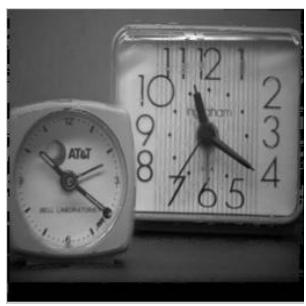

(a)

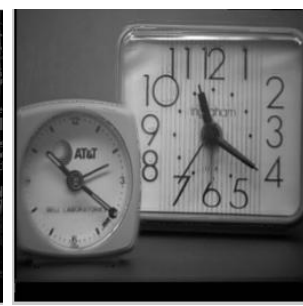

(b)

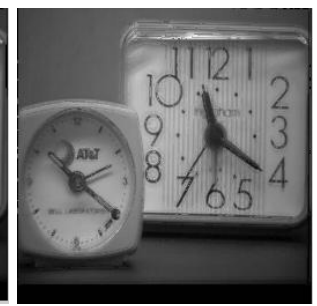

(c)

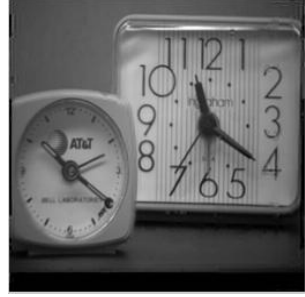

(d)

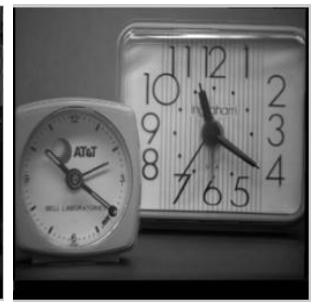

(e)

Fig.7. Experimental results of Image fusion for Clock Image - Fused Image using (a). Variance, (b). Energy gradient, (c). Bilateral sharpness, (d). Wavelet based statistical sharpness, (e). Proposed method

\section{REFERENCES}

[1] H. Li, B. S. Manjunath and S. K. Mitra, "Multi-sensor image fusion using the wavelet transform", Proceedings of First International Conference on Image Processing, Vol. I, pp. 51-55, 1994.

[2] Jing Tian, Li Chen, Lihong Ma and Weiyu Yu, "Multifocus image fusion using a bilateral gradient based sharpness criterion", Optics Communications, Vol. 284, No. 1, pp. 80-87, 2011.

[3] Jing Tian and Li Chen, "Adaptive multi focus image fusion using a wavelet based statistical sharpness measure", Signal Processing, Vol. 92, No. 9, pp. 2137-2146, 2012.

[4] Wang Zuo-cheng, Xue Li-xia and Zhang Xi-ping, "Research on the edges of Image based on cloud model", The International Archives of the Photogrammetry, Remote Sensing and Spatial Information Sciences, Vol. XXXVII. Part B2, pp. 109-114, 2008.

[5] D. Y. Li, C. Y. Liu and W. Y. Gan, "A new cognitive model: Cloud model”, International Journal of Intelligence Systems, Vol. 24, No. 3, pp. 357-375, 2009.

[6] H. J. Wang and Y. Deng, "Spatial clustering method based on cloud model", Proceedings of the IEEE Fourth International Conference on Fuzzy Systems and Knowledge Discovery, Vol. 2, pp. 272-276, 2007.

[7] Y. L. Qi, "Classification for trademark image based on normal cloud model", Proceedings of the IEEE
International Conference on Information Management, Innovation Management and Industrial Engineering, Vol. 3, pp. 74-77, 2009.

[8] H. Chen and B. Li, "Qualitative rules mining and reasoning based on cloud model", Proceedings of the IEEE International Conference on Software Engineering and Data Mining, pp. 523-526, 2010.

[9] K. Qin, K. Xu, Y. Du and D. Y. Li, "An image segmentation approach based on histogram analysis utilizing cloud model", Proceedings of the IEEE Seventh International Conference on Fuzzy Systems and Knowledge Discovery, Vol. 2, pp. 524-528, 2010.

[10] Y. Q. Shi and X. C. Yu, "Image segmentation algorithm based on cloud model the application of fMRI", Proceedings of the IEEE International Conference on Intelligent Computation Technology and Automation, Vol. 2, pp. 136-140, 2008.

[11] Y. Gao, "An optimization algorithm based on cloud model", Proceedings of the IEEE International Conference on Computational Intelligence and Security, Vol. 2, pp. 8487, 2009.

[12] Marta Mrak, Sonja Grgic and Mislav Grgic, "Picture Quality Measures in Image Compression Systems", Proceedings of the IEEE Region 8 EUROCON'03, Vol. 1, pp. 233-237, 2003.

[13] Zhou Wang and Alan C. Bovik, "A Universal Image Quality Index", IEEE Signal Processing Letters, Vol. 9, No. 3, pp. 81-84, 2002. 